\title{
Oak Ridge Research Reactor Quarterly Report October, November, and December of 1976
}
S. S. Hurt, III
E. D. Lance 


\section{DISCLAIMER}

This report was prepared as an account of work sponsored by an agency of the United States Government. Neither the United States Government nor any agency Thereof, nor any of their employees, makes any warranty, express or implied, or assumes any legal liability or responsibility for the accuracy, completeness, or usefulness of any information, apparatus, product, or process disclosed, or represents that its use would not infringe privately owned rights. Reference herein to any specific commercial product, process, or service by trade name, trademark, manufacturer, or otherwise does not necessarily constitute or imply its endorsement, recommendation, or favoring by the United States Government or any agency thereof. The views and opinions of authors expressed herein do not necessarily state or reflect those of the United States Government or any agency thereof. 


\section{DISCLAIMER}

Portions of this document may be illegible in electronic image products. Images are produced from the best available original document. 


\begin{tabular}{|c|}
\hline Printed in the United States of America. Available trom \\
National Technical Information Service \\
U.S. Department of Commerce \\
5285 Port Royal Road, Springfield, Virginia 22161 \\
Price: Printed Copy $\$ 4.00$ Microfiche $\$ 3.00$ \\
\hline
\end{tabular}

This report was prepared as an account of work sponsored by the United States Government. Neither the United States nor the Energy Research and Development Administration/United States Nuclear Regulatory Commission, nor any of their employees, nor any of their contractors, subcontractors, or their employees, makes any warranty, express or implied, or assumes any legal liability or responsibility for the accuracy, completeness or usefulness of any information, apparatus, product or process disclosed, or represents that its use would not infringe privately owned rights. 
Contract No. W-7405-eng-26

OPERATIONS DIVISION

OAK RIDGE RESEARCH REACTOR QUARTERLY REPORT

OCTOBER, NOVEMBER, AND DECEMBER OF 1976

S. S. Hurt, III

E. D. Lance

Date Published - June 1977

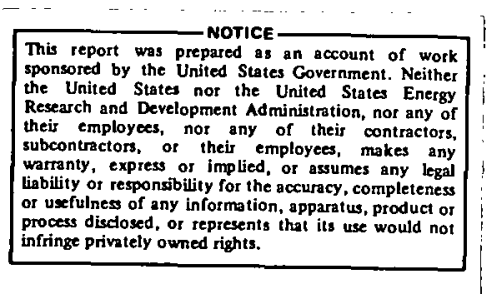

NOTICE This document contains information of a preliminary nature.

It is subject to revision or correction and therefore does not represent a

final report.

OAK RIDGE NATIONAL LABORATORY

Oak Ridge, Tennessee 37830

operated by

UNION CARBIDE CORPORATION

for the

ENERGY RESEARCH AND DEVELOPMENT ADMINISTRATION 


\section{THIS PAGE}

\section{WAS INTENTIONALLY LEFT BLANK}


Summary . . . . . . . . . . . . . . . . . . . . 1

Operations ....................... . . . . 1

Fuel Usage and Inventory . . . . . . . . . . . . . 3

Shutdowns and Power Reductions .. . . . . . . . . . . 4

Instrumentation and Reactor Controls . . . . . . . . . 6

Process System and Mechanical Components . . . . . . . . 6

Experiment Facilities and Core Changes . . . . . . . . . 6

Distribution. . . . . . . . . . . . . . . . . . 21 
SUMMARY

The ORR operated at an average power level of $29.3 \mathrm{Mw}$ for $20.1 \%$ of the time during October, November, and December of 1976. The time operating (\%) is low due to the reduction of operating time to a level compatible with funding. Six fuel elements were declared spent (57.6\% burnup) during the quarter, while no new elements were placed in service.

The reactor was shut down on seven occasions, one of which was unscheduled. Reactor downtime needed for refueling, maintenance and checks was quite low, with the reactor remaining available for operation $92.0 \%$ of the time.

Maintenance activities, both mechanical and instrument, were essentially routine in nature.

OPERATIONS

The basic operating data presented in Table 1 indicates that the ORR operation for the quarter was normal as compared with recent experience. Details relative to cycles of operation during the quarter are given in Tabie 2. 
Table 1. ORR Basic Operating Data

October, November, and December of 1976

\begin{tabular}{|c|c|c|c|c|}
\hline & $\begin{array}{l}\text { This } \\
\text { Quarter }\end{array}$ & $\begin{array}{c}\text { Last } \\
\text { Quarter }\end{array}$ & $\begin{array}{l}\text { Calendar } \\
\text { Year } 1976 \\
\end{array}$ & $\begin{array}{l}\text { Calendar } \\
\text { Year } 1975 \\
\end{array}$ \\
\hline Total energy, Mwd & 554.3 & $1,394.7$ & $6,638.2$ & $9,282.5$ \\
\hline $\begin{array}{l}\text { Average power } \\
\text { Mw/operating hr }\end{array}$ & 29.3 & 29.8 & 29.7 & 29.8 \\
\hline T1me operating, \% & $20.5 *$ & $50.9 *$ & $61.1 *$ & 85.6 \\
\hline Availahility, \% & 92.0 & 95.5 & 92.0 & 00.9 \\
\hline $\begin{array}{l}\text { Reaclur waler radio- } \\
\text { activity } \frac{\mathrm{c} / \mathrm{min}}{\mathrm{m} 1} \text { (av) }\end{array}$ & 25,400 & 28,300 & 27,800 & 29,725 \\
\hline $\begin{array}{l}\text { Pool water radio- } \\
\text { activity, } \frac{\mathrm{c} / \mathrm{min}}{\mathrm{ml}} \text { (av) }\end{array}$ & 570 & 590 & 588 & 647 \\
\hline $\begin{array}{l}\text { Reactor water resis- } \\
\text { tivity, ohm-cm (av) }\end{array}$ & $1,676,000$ & $1,234,000$ & $1,159,000$ & 902,750 \\
\hline $\begin{array}{l}\text { Hool water resis- } \\
\text { tivity, ohm-cm (av) }\end{array}$ & $1,325,000$ & 784,000 & 902,000 & $78.3,250$ \\
\hline Fuel elements depleted & 6 & 1 & 18 & 84 \\
\hline $\begin{array}{l}\text { Average burnup of fuel } \\
\text { eleftents depleted, } \%\end{array}$ & 57.6 & 62.4 & $56.9 * *$ & 52.3 \\
\hline Shim rods depleted & 0 & 2 & 8 & 14 \\
\hline $\begin{array}{l}\text { Average burnup of shim } \\
\text { rods depleced, } \%\end{array}$ & $\dot{0}$ & 69.0 & 65.1 & 64.2 \\
\hline Radioisotope samples & 0 & 2 & 4 & 7 \\
\hline Research samples & 1 & 0 & 7 & 26 \\
\hline
\end{tabular}

*Time operating (\%) low due to reduced leve $\lambda$ of funding.

**Burnup (\%) greater due to lowering of the $235 \mathrm{U}$ weight of the fuel element to $\sim 100$. gm before declaring an element depleted. 
Table 2. Cycles of Operation

\begin{tabular}{ccccc}
\hline Cycle No. & Date Begun & Date Ended & $\begin{array}{c}\text { Accumulated Energy } \\
\text { Mwd }\end{array}$ \\
\hline \multirow{2}{*}{134} & September 13,1976 & November 5, 1976 & $355.1 *$ \\
135 & November 15, 1976 & December 31, 1976 & 306.3 \\
\hline *247.97 Mwd this quarter. &
\end{tabular}

FUEL USAGE AND INVENTORY

Six fuel elements were declared spent during the quarter with an average burnup of 57.6 percent. Thirteen fuel elements and four shim rod elements were shipped to the Savannah River Plant for chemical separation. other details of fuel usage and inventory may be found in Tables 1 and 3 .

Table 3. ORR Fuel Status

\begin{tabular}{|c|c|c|c|c|}
\hline & $\begin{array}{l}\text { This } \\
\text { Quarter }\end{array}$ & $\begin{array}{l}\text { Last } \\
\text { Quarter } \\
\end{array}$ & $\begin{array}{l}\text { Calendar } \\
\text { Year } 1976 \\
\end{array}$ & $\begin{array}{l}\text { Calendar } \\
\text { Year } 1975\end{array}$ \\
\hline $\begin{array}{l}\text { Depleted fuel elements trans- } \\
\text { ferred for chemical separa- } \\
\text { tion }\end{array}$ & 13 & 13 & 71 & 138 \\
\hline $\begin{array}{l}\text { Average percent burnup of fuel } \\
\text { elements transferred }\end{array}$ & 55.2 & 33.8 & 52.8 & 49.5 \\
\hline $\begin{array}{l}\text { New fuel elements placed in } \\
\text { service }\end{array}$ & 0 & 5 & 58 & 104 \\
\hline $\begin{array}{l}\text { New fuel elements available } \\
\text { for use }\end{array}$ & $63 *$ & 63 & & \\
\hline $\begin{array}{l}\text { Depleted shim rods transferred } \\
\text { for chemical separation }\end{array}$ & 4 & 4 & 14 & 18 \\
\hline $\begin{array}{l}\text { Average percent burnup of shim } \\
\text { rods transferred }\end{array}$ & 64.4 & 63.0 & 64.2 & 64.5 \\
\hline New shims placed in service & 0 & 2 & 8 & .14 \\
\hline New shim rods available for use & 2 & 2 & & \\
\hline
\end{tabular}


Reactor downtime (power level $<\mathrm{N}_{\mathrm{L}}$ ) totaled approximately 1,756 hours. A summary of the shutdowns is given in Table 4, and details of each are contained in Table 5. Table 6 offers information relative to the unscheduled shut down.

Table 4. Sumary of Shutdowns

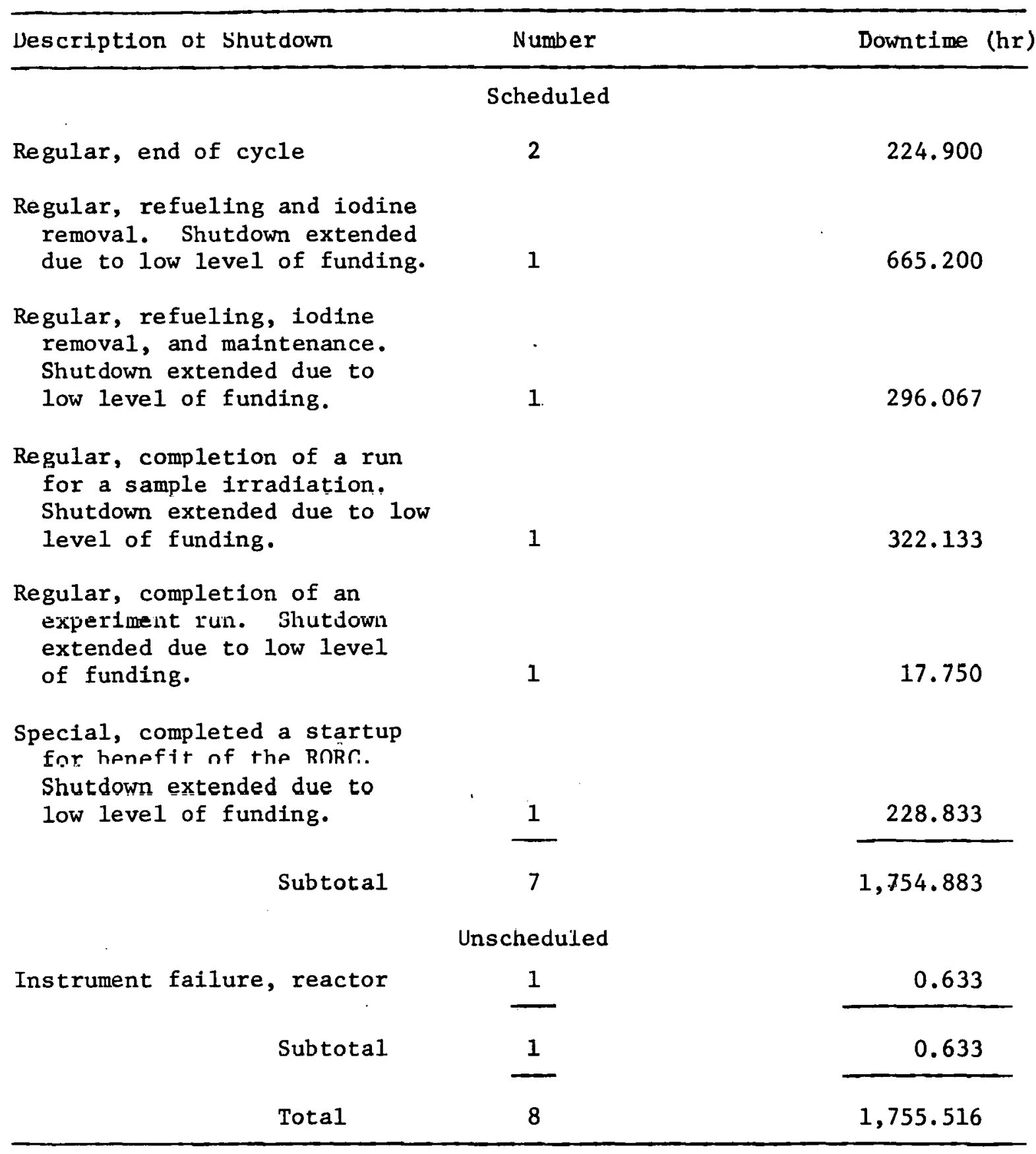


Table 5. ORR Scheduled Shutdowns, Detail

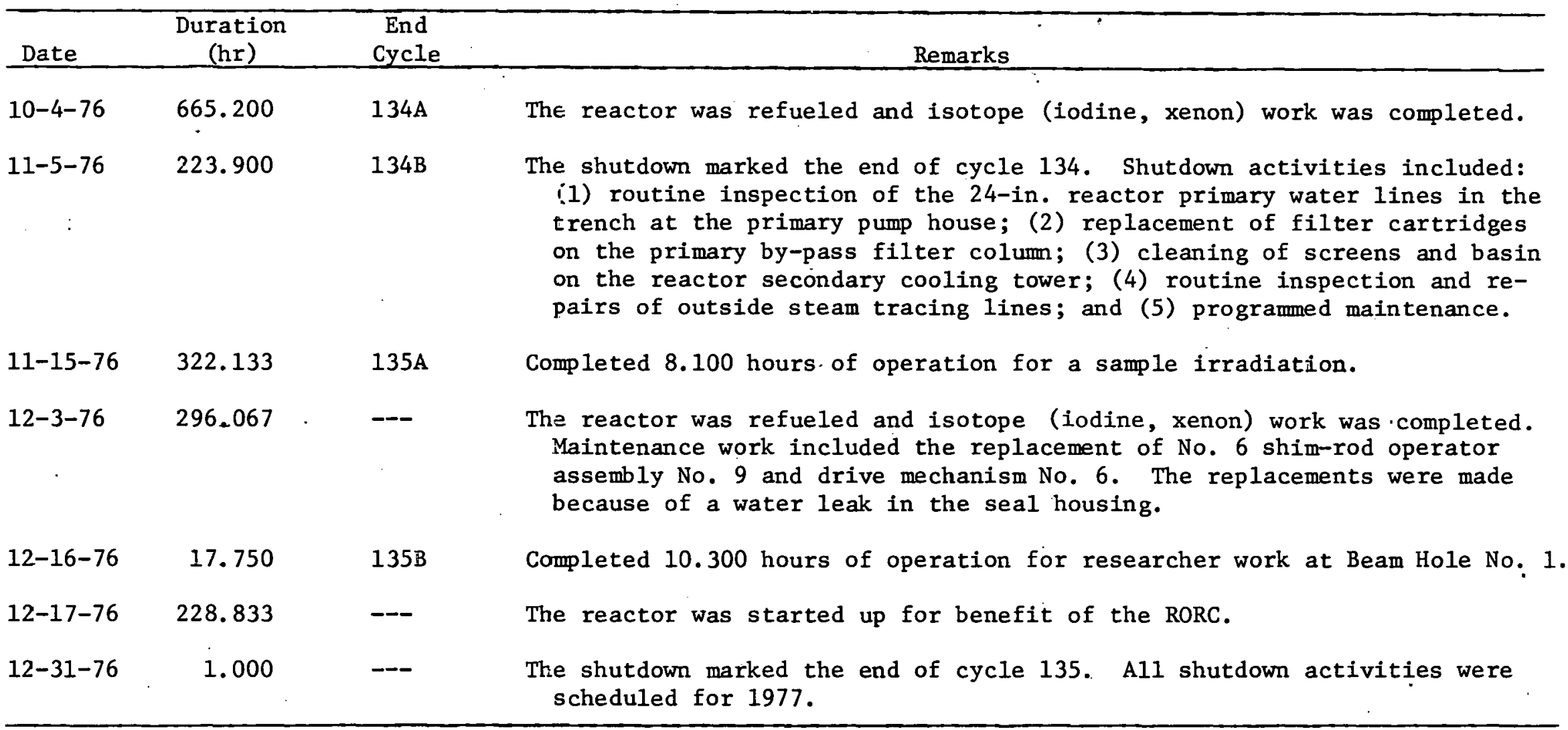

Table 6. ORR Unscheduled Shutdowns, Details

\begin{tabular}{llll}
\hline Date & $\begin{array}{c}\text { Duration } \\
(\mathrm{hr})\end{array}$ & $\begin{array}{c}\text { End } \\
\text { Cycle }\end{array}$ & Remarks \\
\hline $12-16-76$ & 0.633 & -- & $\begin{array}{c}\text { Faulty action (spurious noise spike) from No. 2 Log-N period channel ini- } \\
\text { Eiated a fast scram. }\end{array}$ \\
\hline
\end{tabular}


INSTRUMENTATION AND REACTOR CONTROLS

The performance of the instrumentation for the complex was quite satisfactory and only rather routine maintenance was required as indicated in Table 7. Table 8 reflects the status of the various fission and ionization chambers.

PROCRESS SYSTEM AND MEGHANTRAT, OOMTOLLWT3

Details of maintenance or changes to the process system are given in Table 9, while Table 10 lists the maintenance on the mechanical components. Table 11 summarizes the results of efficiency tests of the various gaseous waste filters.

EXPERIMENT FACILITIES AND CORE CHANGES

Experiment facility usage is given in Table 12. The core configurations used during the quarter are shown in Figure No. 1. 
ORR LATTICE LOADING

For Fue 1 Cycles 134A, 134B, 135A, \& 135B

\begin{tabular}{|c|c|c|c|c|c|c|c|c|}
\hline 1 & 2 & 3 & 4 & 5 & 6 & 7 & .8 & 9 \\
\hline $\mathrm{Be}$ & $\mathrm{Be}$ & $\mathrm{Be}$ & $\mathbf{F}$ & $\mathrm{F}$ & $\mathrm{F}$ & $\mathrm{Be}$ & $\mathrm{Be}$ & $\mathrm{Be}$ \\
\hline $\mathrm{Be}$ & $\mathrm{Be}$ & F & SR & F & SR & $F$ & $\mathrm{Be}$ & $\mathrm{Be}$ \\
\hline $\mathrm{Be}$ & $\mathrm{Be}$ & F & $\mathrm{F}$ & $F$ & $F$ & F & F & $\mathrm{Be}$ \\
\hline $\mathrm{Be}$ & $\mathrm{Be}$ & F & SR & F & SR & $F$ & HT & $\mathrm{Be}$ \\
\hline $\mathrm{Be}$ & $\mathrm{Be}$ & $F$ & $F$ & F & $F$ & $I_{131}$ & $F$ & $\mathrm{Be}$ \\
\hline $\mathrm{Be}$ & $\mathrm{Be}$ & $F$ & SR & $\mathrm{F}$ & SR & $\mathrm{F}$ & SR & $\mathrm{Be}$ \\
\hline $\mathrm{Be}$ & $\mathrm{Be}$ & $\mathrm{Be}$ & $\mathrm{Be}$ & $\mathrm{Be}$ & $\mathrm{Be}$ & $\mathrm{Be}$ & $\mathrm{Be}$ & $\mathrm{Be}$ \\
\hline
\end{tabular}

LATTICE COMPONENT

NUMBER

Fuel

24

Shim Rod (SR)

6

Berylli um (Be)

31

Experiment (E)

0

Iodine sleeve $\left({ }^{131} \mathrm{I}\right)$

Isotope Stringer (Iso)

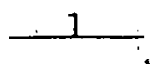

0

Hydraulic Tube (HT)

1

Figure 1. ORR Latt1ce Configuration - October 1, 19.76, Through December 31, 19.76 
Table 7. Maintezance and Changes, Instrumentation and Zontrols

\begin{tabular}{lll}
\hline Date $\quad$ Tromposent & Reas or ctange \\
\hline
\end{tabular}

No. I Courtīng-Rate Channel

12-13-76 No. 1 fission thamber failure
chamber $\quad \begin{gathered}\text { The faulty chamber (TSM-1-52) wes replaced by } \\ \text { chamber TSM-5-67. }\end{gathered}$

No. 2 Courting-Rate Channel

No maintenanze oz zhanges were required.

No. 1 Log-N Channel

No maintenanze or thanges were required.

No. 2 Log-N Channel

No maintenance or changes were required.

No. 1 Safety Channe1

No maintenance or changes were required.

No, 2 safety Channel

No maintenance or changes were required.

No. 3. Safety Channel

No maintenance or changes were required. 
Table 7. (Continued)

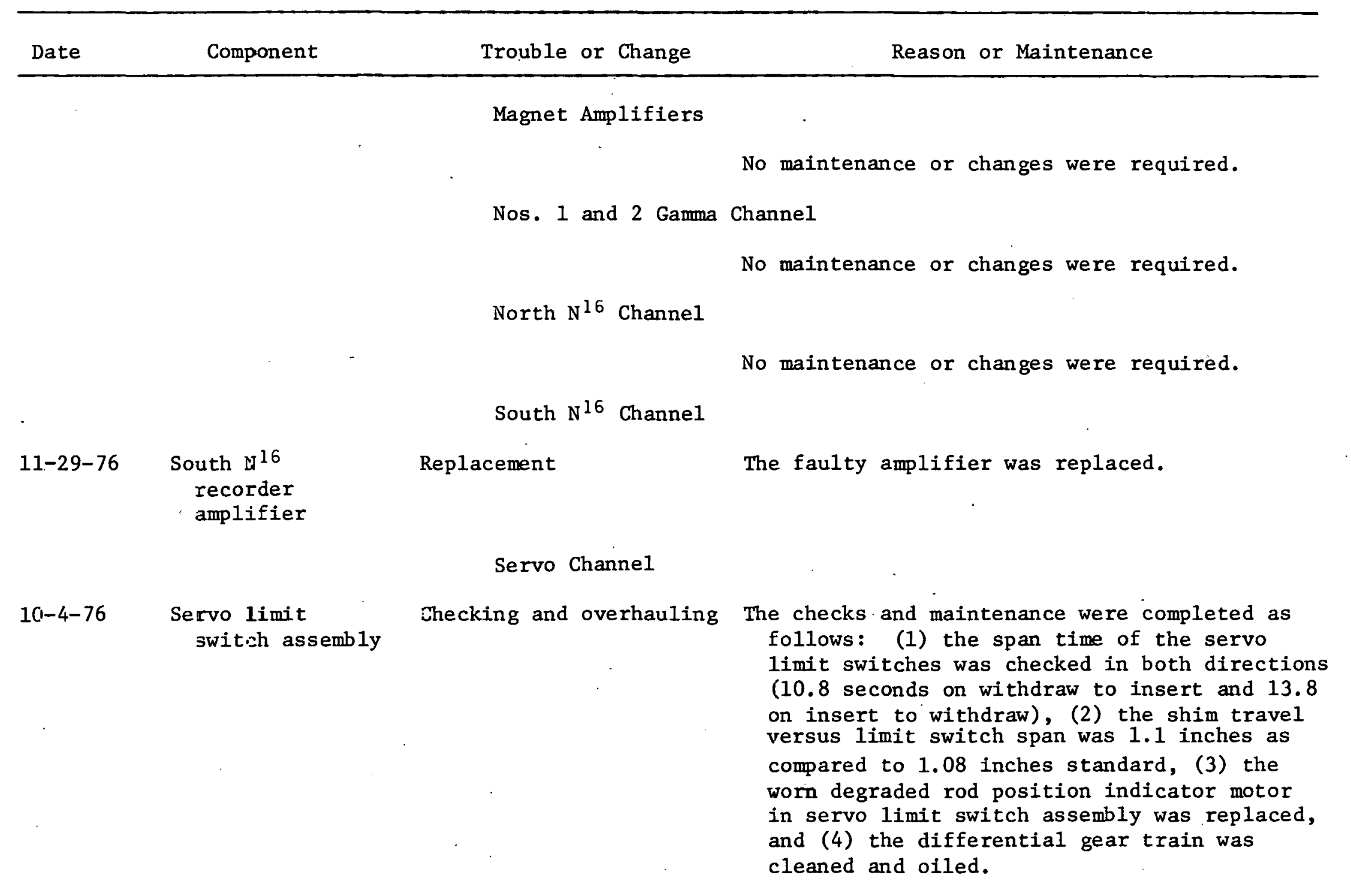


Tab1e 7. (Continued)

\begin{tabular}{|c|c|c|c|}
\hline Date & Component & Trouble or Change & Reason or Maintenance \\
\hline \multicolumn{4}{|c|}{ Reactor Noise Monitor } \\
\hline \multicolumn{4}{|r|}{ No maintenance or changes were required. } \\
\hline \multicolumn{4}{|c|}{ Micrcmicroammeter Channel } \\
\hline \multicolumn{4}{|r|}{ No maintenance or changes were required. } \\
\hline \multicolumn{4}{|c|}{ Process Instrumentation } \\
\hline $10-11-76$ & $\begin{array}{l}\text { Reactor inlet } \\
\text { water temperature } \\
\text { recorder }\end{array}$ & Calibrated & To check calibration. \\
\hline
\end{tabular}


Table 8. Present Status of Ionization Chambers and Fission Chambers

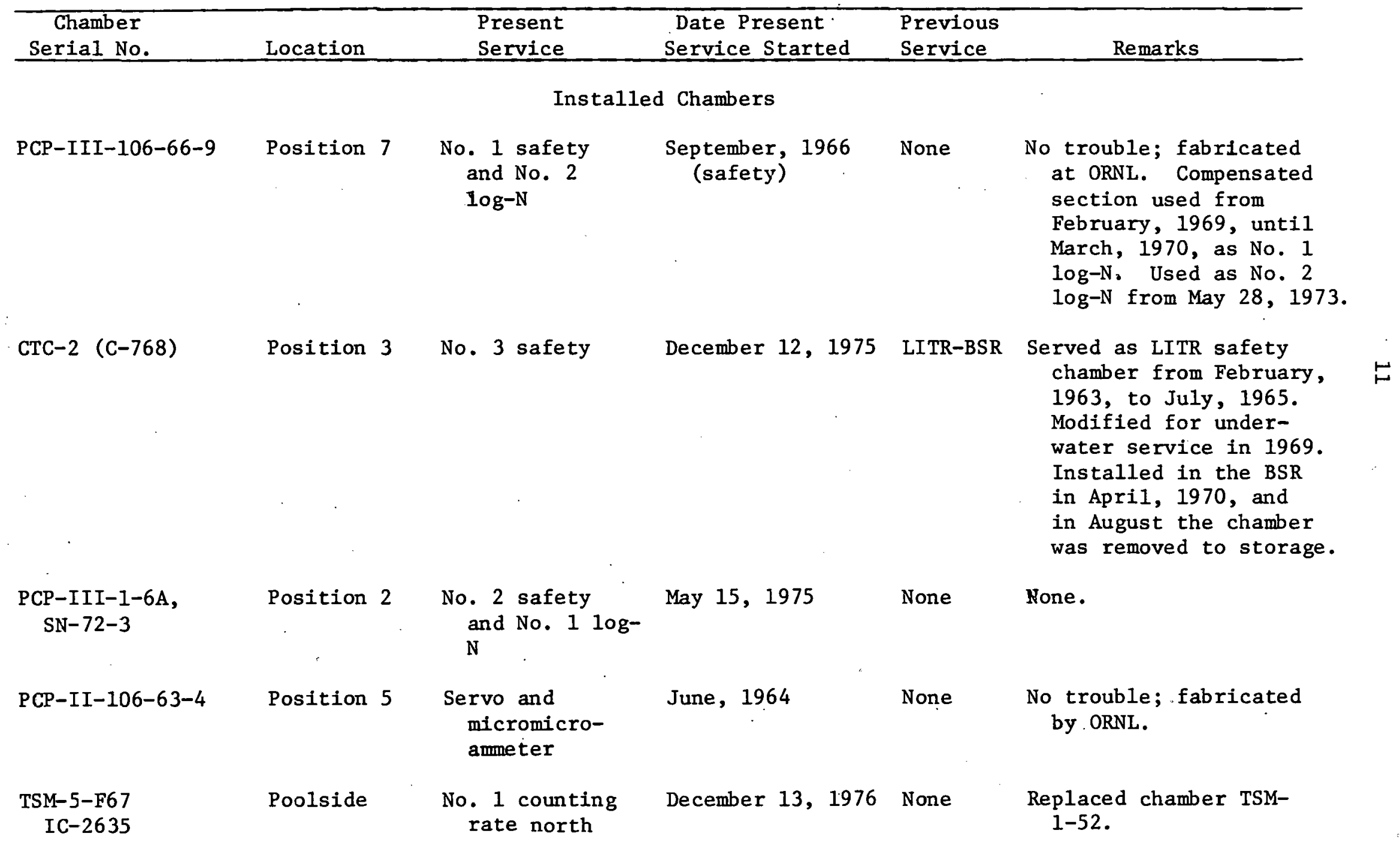


Table 8, (Continued)

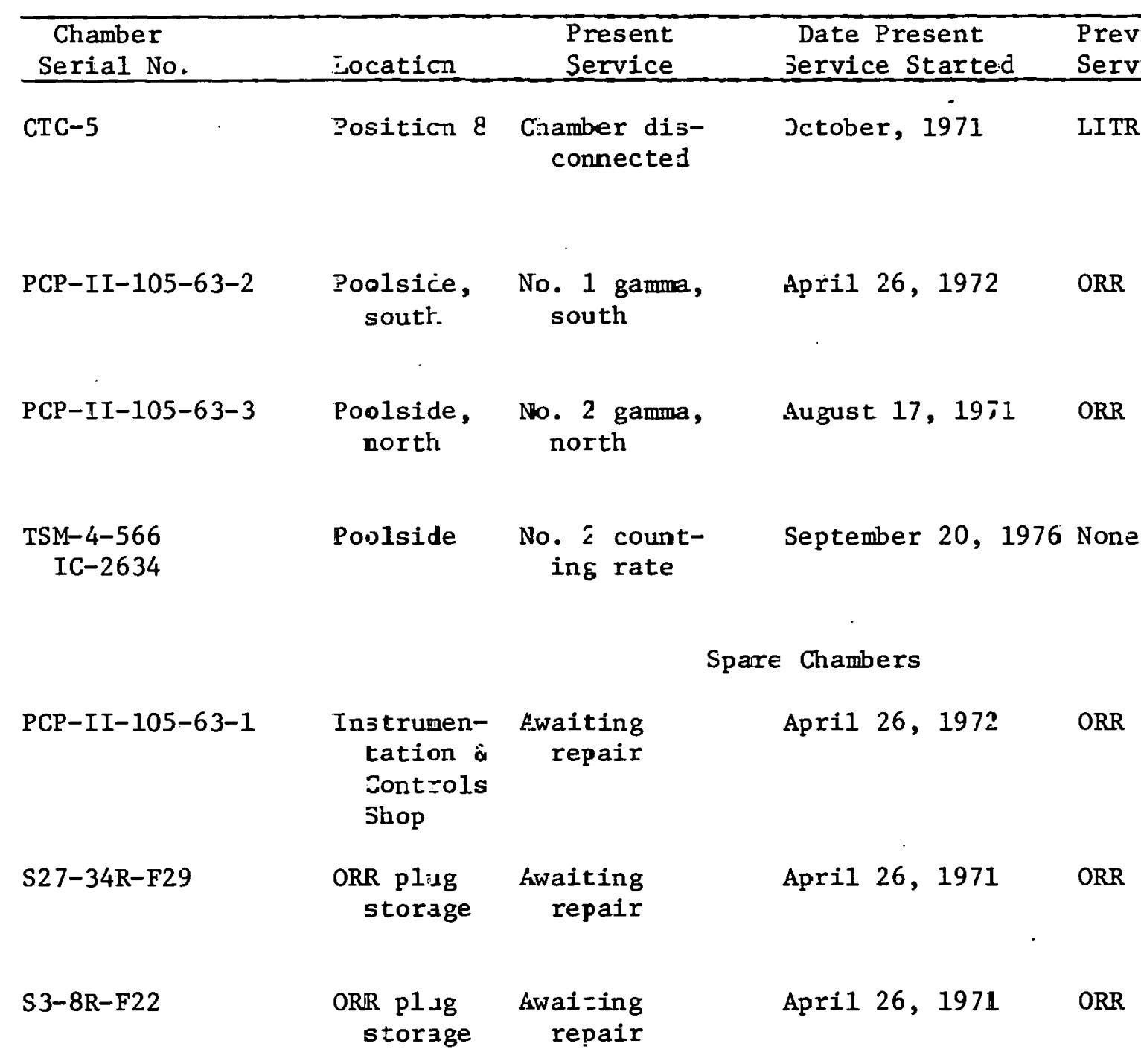

Replaced fission chamber 3-F-29.

Used as No. 2 gamma, north, from April, 1964, until December, 1970.

Used and No. 1 gamma, south, from April, 1964, until March 1970. 
Table 8. (Continued)

\begin{tabular}{|c|c|c|c|c|c|}
\hline $\begin{array}{c}\text { Chamber } \\
\text { Serial No. }\end{array}$ & Location & $\begin{array}{l}\text { Present } \\
\text { Service }\end{array}$ & $\begin{array}{c}\text { Date Present } \\
\text { Service Started }\end{array}$ & $\begin{array}{l}\text { Previous } \\
\text { Service }\end{array}$ & Remarks \\
\hline TSM-1-F52 & $\begin{array}{l}\text { ORR plug } \\
\text { storage }\end{array}$ & $\begin{array}{l}\text { Awaiting } \\
\text { repair }\end{array}$ & $\begin{aligned} & \text { March } 29, 1976 \\
& \text { Removed from } \\
& \text { service } 12 / 13 / 76\end{aligned}$ & ORR & $\begin{array}{l}\text { Used as No. } 2 \text { counting rate } \\
\text { (south) from April } 26, \\
1972 \text {, unti1 October } 24, \\
1972 \text {. Used as No. } 1 \\
\text { counting rate (north) } \\
\text { from March } 29,1976 \text {, un- } \\
\text { til December 13, } 1976 \text {. }\end{array}$ \\
\hline CTC-1 & $\begin{array}{l}\text { Instrumen- } \\
\text { tation \& } \\
\text { Controls } \\
\text { Shop }\end{array}$ & $\begin{array}{l}\text { Awaiting } \\
\text { repair }\end{array}$ & October, 1976 & ORR & $\begin{array}{l}\text { Used as noise monitor from } \\
\text { March, 1962, until } \\
\text { October 1, 1971. }\end{array}$ \\
\hline CTC-4 (C-769) & $\begin{array}{l}\text { ORR plug } \\
\text { storage }\end{array}$ & & & LITR & $\begin{array}{l}\text { Used as No. } 2 \text { safety in the } \\
\text { LITR; modified for ORR- } \\
\text { BSR underwater service. }\end{array}$ \\
\hline TSIL-2-F45 & I\&C shop & $\begin{array}{l}\text { Awaiting } \\
\text { repair }\end{array}$ & August 8,1975 & ORR & $\begin{array}{l}\text { Used as No. } 1 \text { counting rate } \\
\text { from April 26, 1971, un- } \\
\text { til December 5, 1972. } \\
\text { Removed } 3 / 30 / 76 \text { to shop } \\
\text { for repair. }\end{array}$ \\
\hline
\end{tabular}


Tajle 8. (Continued)

\begin{tabular}{|c|c|c|c|c|c|}
\hline $\begin{array}{l}\text { Chamber } \\
\text { Serial No. }\end{array}$ & Locaticn & $\begin{array}{l}\text { Present } \\
\text { Service }\end{array}$ & $\begin{array}{l}\text { Date Present } \\
\text { Service Started }\end{array}$ & $\begin{array}{l}\text { Previous } \\
\text { Service }\end{array}$ & Remarks \\
\hline PCP-II-106-66-3 & $\mathbb{I} \& \mathbb{C}$ Shc.p & $\begin{array}{r}\text { Awaiting } \\
\text { repair }\end{array}$ & $\begin{array}{l}\text { May, } 1974 \text { (safety) } \\
\text { March, 1970, } \\
\text { (log-N). Re- } \\
\text { moved from } \\
\text { December 12, } 1975\end{array}$ & $\begin{array}{l}\text { None } \\
5\end{array}$ & $\begin{array}{l}\text { Fabricated by ORNL. Chamber } \\
\text { Iailed December } 12 \text {, } 1975 . \\
\text { Removed } 9 / 2 / 76 \text { to shop } \\
\text { for repair. }\end{array}$ \\
\hline TSM-3-F29 & I\&C Shop & $\begin{array}{l}\text { Awaiting } \\
\text { refair }\end{array}$ & & ORR & $\begin{array}{l}\text { Used as No. } 2 \text { counting =ate } \\
\text { from October } 24,1972 \text { un- } \\
\text { til September } 20,1975 \text {. }\end{array}$ \\
\hline
\end{tabular}


Table 9. Process Systems, Maintenance and Changes

Date Component $\quad$ Remarks

\section{Reactor Primary Water System}

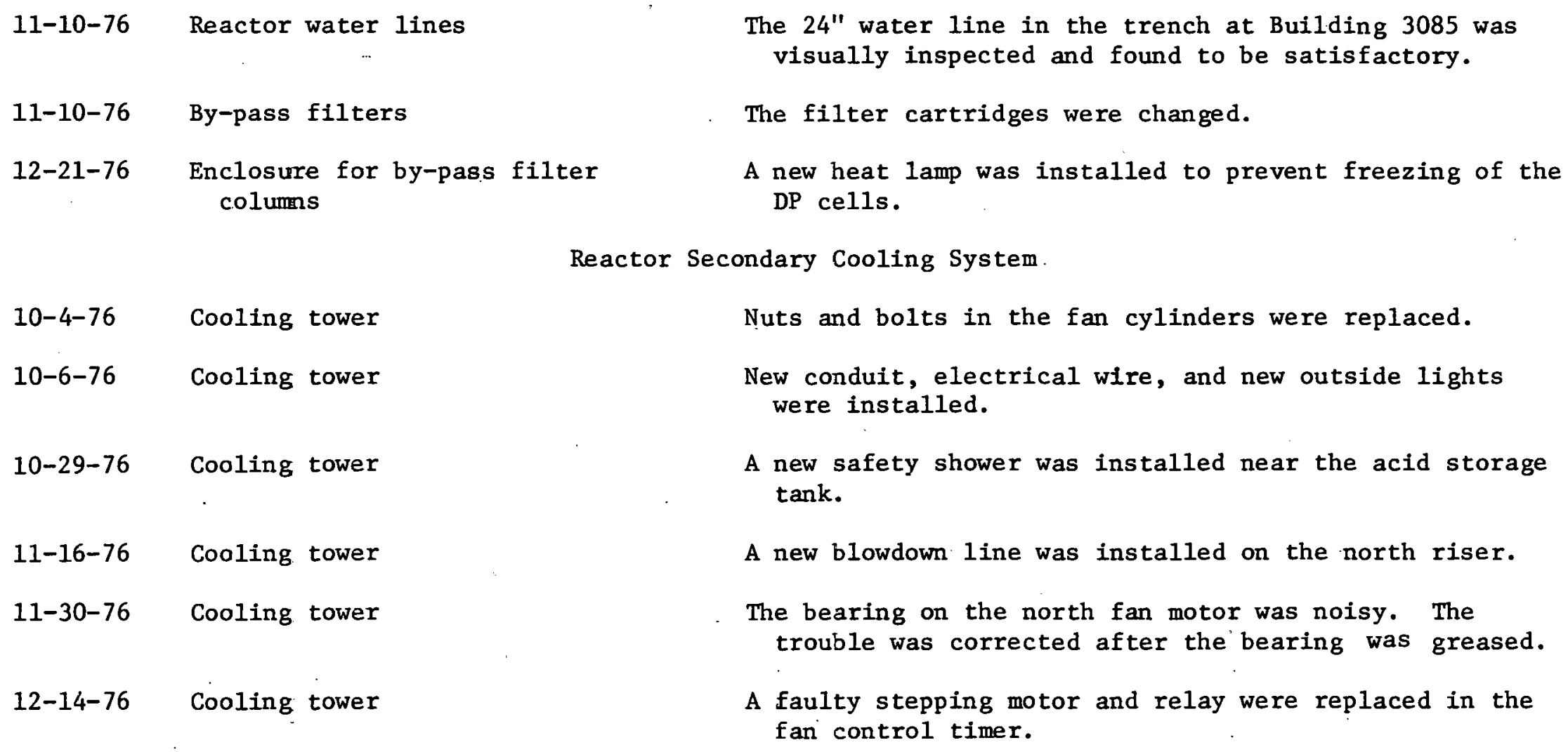


Table 9. (Continued)

Date $\quad$ Component $\quad$\begin{tabular}{c} 
Remeris \\
\hline
\end{tabular}

Pool Frimary Cooling System

10-7-76 Deminera-izeranion colinm

10-27-76 Demineralizer
The cover to the resin colnm was removed and reinstalled after all depleted resin ras removed and new resin installed.

The flange on valve $\mathrm{P}-23$, was ightened to stop a water leak.

Emergency Ccoling System

Functional tests of equipneat and alarms confirmed that the systems were in saistactory condition.

A faulty high voltage relay wes replaced.

\section{Electrücal Systems}

$10-27-76$

\section{Control room instrument cabinet 'B"}

$11-9-76$

Miscellanecus breaker panels for electrïc meters, dc batteries, batter racks, and control roon instrunert cabinets

A faulty exhaust fan was replaced.

Routine end-of-cycle inspection and cleaning were performed. Dust filters for the control room instrument cabinets were changed as needed, and programmed maintenance vas completed.

\section{Emergenzy Elec:rical System}

11-8-76 Diesel generatcr

The routine end-of-cycle lcad test was performed with satisfactory resuits. One battery on the - generator was replaced. 
Table 10. Reactor Mechanical Components, Maintenance and Changes

\begin{tabular}{lcc}
\hline Date & Component & Remarks \\
\hline 10-11-76 North hold-down arm & $\begin{array}{c}\text { Hold-down arm No. } 3 \text { was replaced with No. } 4 \text { hold-down arm which was a } \\
\text { rebuilt unit. }\end{array}$ \\
$\begin{array}{c}\text { No. } 6 \text { shim-rod drive } \\
\text { assembly }\end{array}$ & $\begin{array}{c}\text { The engagement mechanism, seal housing, operator assembly, drive unit } \\
\text { and recocking mechanism were replaced due to a leak in the seal } \\
\text { housing. }\end{array}$ \\
\hline
\end{tabular}

Table 11. Status of Filters - Gaseous Waste System

\begin{tabular}{|c|c|c|c|c|c|}
\hline $\begin{array}{l}\text { Type } \\
\text { Filter } \\
\end{array}$ & $\begin{array}{c}\text { Bank } \\
\text { Designation }\end{array}$ & $\begin{array}{c}\text { Date } \\
\text { Installed } \\
\end{array}$ & $\begin{array}{c}\text { Date Last } \\
\text { Tested }\end{array}$ & Type Test & $\begin{array}{c}\text { Retention } \\
\text { Efficiency \% }\end{array}$ \\
\hline \multicolumn{6}{|c|}{ Cell Ventilation System } \\
\hline CWS & Overal1* & $\begin{array}{l}\text { North, } 6-20-73 \\
\quad \text { South, 1-12-65 }\end{array}$ & $9-30-76$ & DOP & 99.997 \\
\hline Charcoal & Both banks & $\begin{array}{l}\text { North, } 9-16-74 \\
\quad \text { South, 10-17-67 }\end{array}$ & $9-8-76$ & Elemental Iodine & 99.93 \\
\hline Charcoal & Both banks & $\begin{array}{l}\text { North, } 9-16-74 \\
\text { South, 10-17-67 }\end{array}$ & $9-10-76$ & Methyl Iodide & 41.37 \\
\hline \multicolumn{6}{|c|}{ Pressurizable Off-Gas } \\
\hline Chs's & North & $5-5-75$ & $9-30-76$ & DOP & 99.920 \\
\hline
\end{tabular}

*The CWS filters in the cell vencilation system were checked in series. 
Table 11. (Continued)

\begin{tabular}{|c|c|c|c|c|c|}
\hline $\begin{array}{c}\text { Type } \\
\text { Filter } \\
\end{array}$ & $\begin{array}{c}\text { Bank } \\
\text { Desizmation }\end{array}$ & $\begin{array}{c}\text { Bate } \\
\text { Installec }\end{array}$ & $\begin{array}{c}\text { Date Last } \\
\text { Tested }\end{array}$ & Type Test & $\begin{array}{l}\text { Retention } \\
\text { Efficiency \% }\end{array}$ \\
\hline \multicolumn{6}{|c|}{ Pressurizatle off-Gas } \\
\hline Charcoal & Nertin & $2-26-73$ & $1-28-76$ & Elemental Iodine & 99.940 \\
\hline Charcoal & North & $2-26-73$ & $10-7-75$ & Methyl Icdide & 87.970 \\
\hline CWS & South & $10-18-67$ & $9-30-76$ & DOP & 99.986 \\
\hline Charcoa1 & South & $8-11-75$ & $8-13=75$ & Elenental Iodine & 99.993 \\
\hline Charcoal South & South & $8=11-75$ & $10-8-75$ & Metiny 1 Iodide & 97.48 \\
\hline \multicolumn{6}{|c|}{ Basement Hood Exhaust } \\
\hline CWS & South & $1)-4-76$ & $10-4-76$ & DOP & 99.997 \\
\hline CWS & North & $13-4-76$ & $10-4-76$ & DOP & 99.998 \\
\hline \multicolumn{6}{|c|}{ Normal OEf-Gas } \\
\hline CWS & hest & $2-12=76$ & $9-30-76$ & DOP & 99.998 \\
\hline Charcoal & West & $2-12-76$ & $8-3-76$ & Elemental Iodine & 99.94 \\
\hline CWVS & East & $6-12-75$ & $9-30-76$ & DOF & 99.995 \\
\hline Charcoal & Eas: & $7-29-75$ & $10-27-76$ & Elemental Iodine & 99.26 \\
\hline Charcoal & Wes= & $2-12-76$ & $4-20-76$ & Methyl Iodide & 99.93 \\
\hline Charcoal & Eas: & $7-29-75$ & $4-20-76$ & Methyl Iodide & 94.14 \\
\hline
\end{tabular}


Table 12. Experiment Facility Usage

\begin{tabular}{|c|c|c|c|c|c|}
\hline Facility & $\begin{array}{l}\text { Access } \\
\text { Flange } \\
\end{array}$ & $\begin{array}{c}\text { Date } \\
\text { Installed } \\
\end{array}$ & $\begin{array}{c}\text { Date } \\
\text { Removed } \\
\end{array}$ & Description of Experiment & $\begin{array}{l}\text { Division or } \\
\text { Other Sponsor }\end{array}$ \\
\hline $\mathrm{D}-8$ & $v-3$ & $3-10-67$ & & $\begin{array}{l}\text { Hydraulic tube No. } 2 \text { used for } \\
\text { isotope production and short } \\
\text { term irradiations }\end{array}$ & Operations \\
\hline $\mathrm{E}-7$ & None & $8-4-76$ & & Radioisotopes (iodine $\&$ xenon) & Operations \\
\hline $\mathrm{HB}-2$ & None & $7-58$ & $11-76$ & Neutron diffraction experiments & Solid State Physics \\
\hline $\mathrm{HB}-3$ & None & $11-1-58$ & & Neutron diffraction experiments & Solid State Physics \\
\hline $\mathrm{HB}-4$ & None & $7-58$ & $11-76$ & Neutron diffraction experiments & Chemistry \\
\hline $\mathrm{HB}-6$ & None & & & & Solid States \\
\hline $\mathrm{HN}-3$ & None & $11-59$ & & Activation analysis & Analytical Chemistry \\
\hline $\mathrm{HN}-4$ & None & $12-15-63$ & & $\begin{array}{l}\text { Void determinations in } \\
\text { irradiated materials }\end{array}$ & Solid State Physics \\
\hline $\mathrm{HN}-4$ & None & $12-15-63$ & & $\begin{array}{l}\text { "Fly's Eye" two-dimensional } \\
\text { neutron detector for neutron } \\
\text { diffraction and radiography }\end{array}$ & $\begin{array}{l}\text { Instrumentation and } \\
\text { Controls }\end{array}$ \\
\hline
\end{tabular}


THIS PAGE

\section{WAS INTENTIONALLY LEFT BLANK}


INTERNAL DISTRIBUTION

ORNL/TM-5965

1. M. A. Baker

2. F. T. Binford "

3. R. K. Branam

4. G. H. Burger

5. C. D. Cagle

6. W. R. Casto

7. G. H. Coleman

8. J. A. Conlin

9. J. A. Cox

10. F. L. Culler

11. S. J. Ditto

12. W. A. Duggins

13. E. D. Gupton

14. T. P. Hamrick

15. S. S. Hurt, III

16. J. D. Jenkins

17. L. P. Jernigan

18. E. B. Johnson

19. 0. L. Keller

20. E. M. King

21. E. Lamb

22. E. D. Lance
23. L. C. Lasher

24. D. L. Laughlin

25. R. V. McCord

26. F. H. Neil1

27. T. B. Nixon

28. L. C. Oakes

29. H. Postma

30. M. E. Ramsey

31. J. B. Ruble

32. W. H. Sides

33. T. M. Sims

34. M. J. Skinner

35. J. H. Swanks

36. J. R. Thomas

37. W. E. Thomas

38. K. R. Thoms

39. J. R. Weir, Jr.

40. K. W. West

41-43. Laboratory Records Department

44. Laboratory Records, ORNL R.C.

45-46. Central Research Library

47. Document Reference Section

\section{EXTERNAL DISTRIBUTION}

48. K. M. Akhtar, Head, Reactor Operations Group, Pakistan Institute of Nuclear Science \& Technology, Post Office Nilore, Rawalpindi, PAKISTAN

49-50. M. B. Biles, Director, Division of Safety, Standards, and Compliance, ERDA, Washington, D. C.

51. Eric Blomberg, AB Atomenergi, Studsvik, Nyköping, SWEDEN

52. K. J. Bobin, Centre D'Etude de Energie Nucleaire, S.C.K./C.E.N., Boeretang 200, B-2400 Mo1, BELGIUM

53. Safety and Environmental Control Division, ERDA, Oak Ridge

54. C. Möiler, Atomic Energy Board, Pelindaba, Private Bag X256, Pretoria, SOUTH AFRICA

55. Director, Reactor Division, ERDA, Oak Ridge

56. Kiyoshi Inoue, Hitachi Central Research Laboratory, Kokubunji, Tokyo, JAPAN

57. M. Jacquemain, Head, Technical Department ILL, Institute Max von Laue-Paul Langevin, CEDEX 156, 38 Grenoble-Gare, FRANCE

58. Masanori Kanbara, Head, Reactor Section No. 2, JMTR Division, OARAI Research Establishment, JAERI, JAPAN

59. Wilfried Kru11, Gesellschaft fur Kernenergieverwertung, In Schiffbau und Schiffahrt MBH., 2054 Geesthacht-Tesperhude, Reaktorstrabe-1, WEST GERMAN FEDERAL REPUBLIC

60. O. D. Oner, Head, Reactor Operation Division, Cemece Nuclear Research Center, P.K. 1. Hava Alani, Istanbul, TURKEY

61. G. Rada, Head, Reactor Department, Institute Venezolano de Invest1gaciones Cientificas, Apartado 1827, Caracas, VENEZUELA 
EXTERNAL DISTRIBUTION (continued)

62. Research and Technical Support Division, ERDA, Oak Ridge

63. Robert J. Swanenburg de Veye, Head, Research Reactor Department, Reactor Centrum Nederland, Westerduinweg 3-Petten (N.-H.), THE NETHERLANDS

64-91. Technical Information Center, Oak Ridge

92. R. Wakabayashi, NAIG Research Laboratory, 250 Suehiro-Cho. Kawasaki City, Kanagawa Prefecture, JAPAN

93. K. E. Wirtz, Institute of Technology, Nuclear Research Center, Kar1sruhe, WEST GERMAN FEDERAL REPUBLIC

94. A. C. Wood, A.A.E.C. Research Establishment, New Illawarra Rd., Lucas Hts., N.S.W., MUSTRALIA 